\title{
Can Whole-body MRI Replace CT in Management of Metastatic Testicular Cancer? A Prospective, Non- inferiority Study.
}

\section{Solveig Kärk Abildtrup Larsen ( $\square$ solveig.larsen@auh.rm.dk)}

Aarhus University Hospital: Aarhus Universitetshospital https://orcid.org/0000-0001-5482-7650

\section{Vibeke Løgager}

Herlev Hospital

\section{Catharina Bylov}

Aarhus University Hospital: Aarhus Universitetshospital

\section{Hanne Nellemann}

Aarhus University Hospital: Aarhus Universitetshospital

\section{Mads Agerbæk}

Aarhus University Hospital: Aarhus Universitetshospital

\section{Anne Birgitte Als}

Aarhus University Hospital: Aarhus Universitetshospital

\section{Erik Morre Pedersen}

Aarhus University Hospital: Aarhus Universitetshospital

\section{Research Article}

Keywords: CT (computed tomography), DWI (diffusion-weighted imaging), DWIBS (diffusion-weighted whole-body imaging with background body signal suppression), MRI (magnetic resonance imaging), non-inferiority study, testicular cancer

Posted Date: January 3rd, 2022

DOI: https://doi.org/10.21203/rs.3.rs-1216327/v1

License: (c) (i) This work is licensed under a Creative Commons Attribution 4.0 International License. Read Full License

Version of Record: A version of this preprint was published at Journal of Cancer Research and Clinical Oncology on April 7th, 2022. See the published version at https://doi.org/10.1007/s00432-022-03996-1. 


\section{Abstract}

Purpose Concerns of imaging-related radiation in young patients with high survival rates have increased the use of magnetic resonance imaging (MRI) in testicular cancer (TC) stage I. However, computed tomography (CT) is still preferred for metastatic TC.

The purpose of this study was to compare whole-body MRI incl. diffusion-weighted whole-body imaging with background body signal suppression (DWIBS) with contrast-enhanced, thoracoabdominal CT in metastatic TC.

Methods A prospective, non-inferiority study of 84 consecutive patients (median age 33 years) with newly diagnosed metastatic TC (February 2018 - January 2021). Exclusion criteria were age < 18 years, claustrophobia and MRI contraindications. Patients had both MRI and CT before and after treatment. Anonymised images were reviewed by experienced radiologists.

Lesion malignancy was evaluated on a Likert scale (1 benign - 4 malignant). Sensitivity, specificity, positive predictive value, negative predictive value and accuracy were calculated on patient and lesion level. For non-inferiority testing, the difference in sensitivity between CT and MRI was calculated. The level of significance was set at $5 \%$. ROC curves and interobserver agreement were calculated.

Results On patient level, MRI had 98\% sensitivity and 75\% specificity compared to CT. On lesion level within each modality, MRI had $99 \%$ sensitivity and $78 \%$ specificity, whereas CT had $98 \%$ sensitivity and $88 \%$ specificity. MRI sensitivity was non-inferior to CT (difference $0.57 \%$ (95\% Cl-1.4-2.5\%)). The interobserver agreement was substantial between CT and MRI.

Conclusion MRI with DWIBS was non-inferior to contrast-enhanced CT in detecting metastatic TC disease.

Trial registration www.clinicaltrials.gov NCT03436901, finished July $1^{\text {st }} 2021$.

\section{Introduction}

Testicular cancer (TC) is a common cancer in young men of western societies (Laguna et al. 2020). TC metastases are predominantly located in the retroperitoneum and lungs (Laguna et al. 2020). The treatment of metastatic TC with chemotherapy or radiotherapy is very effective resulting in very high 5-year survival rates of 90-95\% (Laguna et al. 2020).

The surveillance strategy for TC includes clinical examination, serum tumour markers and, not least, regular imaging. European guidelines recommend four contrast-enhanced abdominopelvic computed tomography (CT) scans in the first five years after chemotherapy (Laguna et al. 2020).

The cumulative imaging-related doses in TC is a concern (Tarin et al. 2009; Van Walraven et al. 2011; Silva et al. 2012; Pandharipande et al. 2013) and as a consequence, the use of magnetic resonance imaging (MRI) in follow-up of TC stage I is increasing (Stephenson et al. 2019; Laguna et al. 2020; DaTeCa 2021; SWENOTECA (Swedish and Norwegian Testicular Cancer Group) 2021). Recently, a standardized MRI protocol for follow-up of TC stage I was proposed (SWENOTECA (Swedish and Norwegian Testicular Cancer Group) 2021). Likewise, several guidelines list MRI as an option after chemotherapy (The Danish Health and Medicines Authority 2015; SWENOTECA (Swedish and Norwegian Testicular Cancer Group) 2021). 
The evidence is limited regarding MRI in detection of TC metastasis. However, in a few small studies, the diagnostic accuracy of MRI was similar to results from a systematic review of CT (MRI 75-100\% sensitivity, 89-100\% specificity. CT 66\% sensitivity, 92\% specificity) (Sohaib et al. 2009; Laukka et al. 2020; Pierorazio et al. 2020; Narine et al. 2021). In addition, a prospective, multi-centre RCT on follow-up of seminoma stage I with two CT arms and two MRI arms is in progress (Sohaib et al. 2009).

The use of MRI is limited by high costs and long scan time (Su et al. 2015), but with constant technology advances, image quality is improving and scan time is reducing. For this study, a dedicated non-contrast-enhanced whole-body (WB) MRI protocol with diffusion-weighted whole-body imaging with background body signal suppression (DWIBS) was used with approx. 30 minutes scan time.

The aim of this prospective, non-inferiority-study, was to study the ability of WB-MRI with DWIBS to replace standard contrast-enhanced, thoracoabdominal CT in metastatic TC. This study covered the pre-treatment staging scan and the first post-treatment evaluation scan.

\section{Patients And Methods Study design}

A prospective, non-inferiority study of MRI with DWIBS (new test) compared to contrast-enhanced CT (reference test) was designed. A power analysis was performed for a paired design (patients were their own controls) with binary outcome (metastases or no metastases on a lesion basis) (Liu et al. 2002). Using $80 \%$ CT sensitivity and $74 \%$ MRI sensitivity at $5 \%$ 's level of significance and $80 \%$ power, a sample size of 235 lesions was calculated.

\section{Patients}

Consecutive patients initiating treatment for metastatic TC were included at our institution from February 2018 to January 2021. Patients with primary metastatic TC, relapse during follow-up or pure serologic relapse were included. Patients with age $<18$ years, claustrophobia or MRI contraindications were excluded.

The patients were approached with both oral and written study information at the oncological department after diagnosis of metastatic TC. Written consent was obtained after at least 24 hours of consideration unless immediate treatment was imperative.

Treatment was in accordance with national guidelines and was not altered by study participation (DaTeCa 2021).

\section{Imaging}

All patients had CT and MRI of the thorax, abdomen and pelvis performed prior to treatment initiation and after treatment (three weeks after last chemotherapy or three months after completing radiotherapy in accordance with national guidelines (The Danish Health and Medicines Authority 2015; DaTeCa 2021). After study participation, patients returned to standard follow-up with CT.

Clinical CTs were used as much as possible. If a recent contrast-enhanced thoracoabdominal CT was available, no additional CT was performed. Some diagnostic contrast-enhanced CTs were retrieved from pre-treatment PET-CT.

A dedicated WB-MRI protocol was developed based on the existing clinical MRI protocol of the retroperitoneum and pelvis for follow-up of TC stage I (Larsen et al. 2020) with extension to the upper abdomen and thorax. 
A 1.5T MRI system was used (Ingenia, release 5.3 with DDAS spectrometer, Philips Medical Systems, Best, The Netherlands) with the built-in posterior coil, dS head-neck coil and flex coverage anterior coil.

The same non-contrast-enhanced protocol was used throughout the study (Table 1): T1 (coronal), T2 (axial), freebreathing WB-DWIBS (axial) and low-resolution, respiratory-triggered, DWIBS b200 (lungs and liver, axial). Coverage was identical for T1, T2 and WB-DWIBS (from neck to groin). The total table time incl. preparation, pre-scans, breath holds and respiratory triggering was approx. 30 minutes. All scans were anonymised using OsiriX (version 10.0, Pixmeo, Bernex, Switzerland).

Table 1

Magnetic resonance imaging parameters at 1.5T (Philips Ingenia).

\begin{tabular}{|c|c|c|c|c|}
\hline Sequence & WB-DWIBS & DWIBS b200 & T2 & $\mathrm{T} 1$ \\
\hline Imaging mode & EPI SE SS & EPISE SS & SS PSS & VISTA PSS 3D \\
\hline $\begin{array}{l}\text { Duration per station } \\
\text { (min:s) }\end{array}$ & $2: 17$ & $1: 03^{*}$ & $0: 14$ & 0:15 \\
\hline No. of images & 144 & 62 & 144 & 181 \\
\hline Resolution (mm) & $4 \times 4 \times 6$ & $5 \times 5 \times 5$ & $1.5 \times 1.5 \times 6$ & $1.7 \times 1.7 \times 3.4$ \\
\hline Plane & Axial & Axial & Axial & $\begin{array}{l}\text { 3D, coronal } \\
\text { reconstruction }\end{array}$ \\
\hline \multirow[t]{3}{*}{ Stations } & Thorax & Thorax/liver & Thorax & Thorax \\
\hline & Abdomen & & Abdomen & Abdomen \\
\hline & Pelvis & & Pelvis & Pelvis \\
\hline Echo time (ms) & 68 & 49 & 80 & 46 \\
\hline Repetition time (ms) & 6531 & 1371 & 7158 & 310 \\
\hline Multi-Band & No & $\begin{array}{l}\text { Yes, acceleration factor } \\
2\end{array}$ & - & - \\
\hline Respiration & $\begin{array}{l}\text { Free } \\
\text { breathing }\end{array}$ & Respiratory trigger & $\begin{array}{l}\text { Breath } \\
\text { hold }\end{array}$ & Breath hold \\
\hline Fat saturation & STIR & STIR & - & - \\
\hline B-values (s/mm²) & $0,50,800$ & 200 & - & - \\
\hline NSA & $2,2,4$ & 2 & - & - \\
\hline LOVA ADC & On & Off & - & - \\
\hline SENSE & 2 & 2 & 2 & 8 \\
\hline
\end{tabular}

CTs were evaluated in consensus by two oncological radiologists (18/30 years' experience). MRIs were evaluated independently by two oncological radiologists (14/23 years' experience) with subsequent consensus reading. Except for the diagnosis of metastatic TC, no clinical information was provided. To mimic clinical routine, the pre-treatment scan was evaluated alone first, whereafter the post-treatment scan was evaluated side-by-side with the pre-treatment scan. 
The five largest lesions were recorded in eight anatomical regions: lungs, supraclavicular, mediastinum, retroperitoneum along the aorta, retroperitoneum along the iliac vessels, groin, other lymph node metastases and other metastases. For each lesion, anatomical position, pre- and post-treatment description, pre- and post-treatment diameter (shortest lymph node axis on CT/T2), and pre- and post-treatment Likert score of malignancy were recorded. Presence of other pathological findings was recorded.

All data were managed using REDCap (Vanderbilt University, Tennessee, USA).

\section{Statistical analysis}

A Likert scale was constructed (1 benign, 2 probably benign, 3 probably malignant, 4 malignant) (Norman 2010). Likert 1-2 was considered benign and Likert 3-4 was considered malignant. A category representing uncertainty was avoided. The post-treatment Likert score included evaluation of treatment response in each lesion using each patient as their own control.

The reference for malignancy was defined as Likert 3-4 on post-treatment CT. The presence of malignancy was evaluated on patient and lesion level.

Sensitivity, specificity, positive predictive value, negative predictive value and accuracy were calculated with $95 \%$ confidence intervals $(\mathrm{Cl})$. On a lesion level, data were adjusted for clustering in patients. Lesions too small for measurement were included if measured on the other modality.

ROC curves were constructed using different cut-off Likert scores for malignancy.

For non-inferiority testing, MRI and CT results could not be intermingled. Thus the pre-treatment was compared to posttreatment scan in each modality as reference. The difference in sensitivity was calculated with two-sided $95 \% \mathrm{Cl}$ (Liu et al. 2002). The level of significance was $5 \%$.

Interobserver agreement was estimated using kappa statistics (Norman 2010) in describing each lesion as benign (Likert 1-2) or malignant (Likert 3-4) after review of both scans.

\section{Results}

Ninety patients were included, but one tumour was reclassified as rhabdomyosarcoma and one suspicion of relapse was dropped. Four patients dropped out due to treatment side effects (tumour burden and comorbidity did not differ from remaining patients).

In the final analysis, 84 patients were included: 40 seminomas (S) and 44 non-seminomas (NS). The median age was 33 years [IQR 28-43 years], median weight was $85 \mathrm{~kg}$ [IQR 78-95 kg] and median height was $181 \mathrm{~cm}$ [IQR 180-185 cm].

Thirty-seven patients had primary metastatic TC (S 11, NS 26) and 47 relapsed during stage I surveillance (S 29, NS 18). Biopsies confirmed diagnosis in 47 patients (S 32, NS 15) and were inconclusive in two patients (NS 2). In 35 patients, no biopsy was performed (S 8, NS 27). The median time from diagnosis to relapse was seven months [IQR 414 months] (S eight months [IQR 6-15 months], NS four months [IQR 2-10 months]). Sixty-seven patients received chemotherapy (S 23, NS 44) and 17 received radiotherapy (S 17). Eleven patients were operated for residual tumour after chemotherapy (8 teratomas, 1 embryonal carcinoma, 1 rhabdomyosarcoma and 1 pure necrosis).

By September $13^{\text {th }} 2021$, two patients had died (of pneumonia after 5 months and of rhabdomyosarcoma-component after 25 months). Two patients emigrated. The median follow-up time was 29 months [IQR 18-41 months]. 
Pre-treatment MRI and CT were a median of 5 days apart [IQR 0-18 days] and the post-treatment MRI and CT were a median of 0 days apart [IQR 0-0 days]. The median time between pre- and post-treatment MRI was 63 days [IQR 59-84 days] (chemotherapy 63 days [IQR 58-69 days], radiotherapy 116 days [IQR 112-122 days]). Twenty-two CTs were derived from PET-CT. No adverse reactions to imaging occurred. Only few comorbidities were visible on imaging.

On patient level, malignancy was found in 80 patients by CT and in 79 patients by MRI. Compared to CT, MRI had $98 \%$ sensitivity (95\% Cl 91-100\%) and 75\% specificity (95\% Cl 19-99\%). One MRI was false positive and two MRIs were false negative. Details table $2 \mathrm{~A}$ and 3 , image examples Fig 1.

Table 2

Contingency tables of results with sensitivity, specificity, positive predictive value (PPV), negative predictive value (NPV) and accuracy incl. 95\% confidence intervals.

Computed tomography (CT). Magnetic resonance imaging (MRI). 


\begin{tabular}{|c|c|c|c|c|c|}
\hline \multicolumn{6}{|c|}{ A Patient level, post-treatment MRI vs. post-treatment CT } \\
\hline & \multicolumn{3}{|c|}{ Post-treatment CT } & \multirow{2}{*}{$\begin{array}{l}\text { Sensitivity } \\
\text { Specificity }\end{array}$} & \multirow{2}{*}{$\begin{array}{l}98 \%(91-100 \%) \\
75 \%(19-99 \%)\end{array}$} \\
\hline Post-treatment MRI & No malignancy & Malignancy & Sum & & \\
\hline No malignancy & 3 & 2 & 5 & PPV & $99 \%(93-100 \%)$ \\
\hline Malignancy & 1 & 78 & 79 & NPV & $60 \%(15-95 \%)$ \\
\hline Sum & 4 & 80 & 84 & Accuracy & $97 \%(90-99 \%)$ \\
\hline \multicolumn{6}{|c|}{ B Lesion level, pre- vs. post-treatment CT } \\
\hline & \multicolumn{3}{|c|}{ Post-treatment CT } & Sensitivity & $98 \%(96-100 \%)$ \\
\hline Pre-treatment CT & Benign & Malignant & Sum & Specificity & $88 \%(78-95 \%)$ \\
\hline Benign & 60 & 5 & 65 & PPV & $97 \%(94-99 \%)$ \\
\hline Malignant & 8 & 251 & 259 & NPV & $92 \%(83-98 \%)$ \\
\hline Sum & 68 & 256 & 324 & Accuracy & $96 \%(93-98 \%)$ \\
\hline \multicolumn{6}{|c|}{ C Lesion level, pre-vs. post-treatment MRI } \\
\hline & \multicolumn{3}{|c|}{ Post-treatment MRI } & Sensitivity & $99 \%(97-100 \%)$ \\
\hline Pre-treatment MRI & Benign & Malignant & Sum & Specificity & $78 \%(69-86 \%)$ \\
\hline Benign & 78 & 4 & 82 & PPV & $93 \%(89-96 \%)$ \\
\hline Malignant & 22 & 286 & 308 & NPV & $95 \%(88-99 \%)$ \\
\hline Sum & 100 & 290 & 390 & Accuracy & $93 \%(90-96 \%)$ \\
\hline \multicolumn{6}{|c|}{ D Lesion level, post-treatment MRI vs. post-treatment CT } \\
\hline & \multicolumn{3}{|c|}{ Post-treatment CT } & Sensitivity & $96 \%(94-99 \%)$ \\
\hline Post-treatment MRI & Benign & Malignant & Sum & Specificity & $87 \%(69-96 \%)$ \\
\hline Benign & 26 & 8 & 34 & PPV & $98 \%(95-100 \%)$ \\
\hline Malignant & 4 & 200 & 204 & NPV & $77 \%(59-89 \%)$ \\
\hline Sum & 30 & 208 & 238 & Accuracy & $95 \%(91-97 \%)$ \\
\hline \multicolumn{6}{|c|}{ E Patient level, lungs, post-treatment MRI vs. post-treatment CT } \\
\hline & \multicolumn{3}{|c|}{ Post-treatment CT } & Sensitivity & $82 \%(48-98 \%)$ \\
\hline Post-treatment MRI & No malignancy & Malignancy & Sum & Specificity & $88 \%(78-94 \%)$ \\
\hline No malignancy & 26 & 8 & 34 & PPV & $50 \%(26-74 \%)$ \\
\hline Malignancy & 5 & 199 & 204 & NPV & $97 \%(90-100 \%)$ \\
\hline Sum & 31 & 207 & 238 & Accuracy & $87 \%(78-93 \%)$ \\
\hline \multicolumn{6}{|c|}{ F Lesion level, lungs, post-treatment MRI vs. post-treatment CT } \\
\hline & \multicolumn{3}{|c|}{ Post-treatment CT } & Sensitivity & $96 \%(80-100 \%)$ \\
\hline Post-treatment MRI & Benign & Malignant & Sum & Specificity & $50 \%(1.3-99 \%)$ \\
\hline
\end{tabular}




\begin{tabular}{|llllll|}
\hline Benign & 1 & 1 & 2 & PPV & $96 \%(80-100 \%)$ \\
\hline Malignant & 1 & 24 & 25 & NPV & $50 \%(1.3-99 \%)$ \\
\hline Sum & 2 & 25 & 27 & Accuracy & $93 \%(76-99 \%)$ \\
\hline
\end{tabular}

Table 3

Lesion level disagreement between post-treatment magnetic resonance imaging (MRI) and post-treatment computed tomography (CT). Patient level disagreement indicated by *. False positive (FP). False negative (FN). 


\begin{tabular}{|c|c|c|c|c|c|}
\hline No. & & Region & MRI & CT & $\begin{array}{l}\text { Subsequent imaging } \\
\text { (months after diagnosis of metastatic } \\
\text { disease) }\end{array}$ \\
\hline 3 & FP & Lung & Right side, Likert 4 & $\begin{array}{l}\text { Postinfectious, } \\
\text { right side, Likert } 1\end{array}$ & $\begin{array}{l}\text { CT after } 9,16 \text { and } 40 \text { months without signs } \\
\text { of lung metastasis. }\end{array}$ \\
\hline 75 & $\mathrm{FN}$ & Lung & $\begin{array}{l}\text { Left side, } 5 \mathrm{~mm} \text {, } \\
\text { Likert } 4\end{array}$ & $\begin{array}{l}\text { Left side, } 2 \text { mm, } \\
\text { Likert } 2\end{array}$ & $\begin{array}{l}\text { CT after } 5 \text { and } 8 \text { months without signs of } \\
\text { lung metastasis. }\end{array}$ \\
\hline 10 & $\mathrm{FN}$ & $\begin{array}{l}\text { Retroperi- } \\
\text { toneum } \\
\text { (aorta) }\end{array}$ & $\begin{array}{l}\text { Several small, } 6 \\
\text { mm, Likert } 4\end{array}$ & $\begin{array}{l}\text { By left psoas, } 6 \\
\text { mm, Likert } 2\end{array}$ & $\begin{array}{l}\text { Unchanged on CT after 10, } 16 \text { and } 40 \\
\text { months. }\end{array}$ \\
\hline 14 & FN & $\begin{array}{l}\text { Retroperi- } \\
\text { toneum } \\
\text { (aorta) }\end{array}$ & $\begin{array}{l}\text { By vena cava, } 9 \\
\mathrm{~mm} \text {, Likert } 4\end{array}$ & $\begin{array}{l}\text { By vena cava, } 9 \\
\text { mm, Likert } 2\end{array}$ & $\begin{array}{l}\text { CT after } 15 \text { months without signs of } \\
\text { retroperitoneal metastasis. }\end{array}$ \\
\hline 19 & FN & $\begin{array}{l}\text { Retroperi- } \\
\text { toneum } \\
\text { (aorta) }\end{array}$ & $\begin{array}{l}\text { Several small, not } \\
\text { measurable, Likert } \\
3\end{array}$ & $\begin{array}{l}\text { Left side, } 8 \mathrm{~mm} \text {, } \\
\text { Likert } 2\end{array}$ & Unchanged on CT after 10 and 14 months. \\
\hline $23 *$ & FN & $\begin{array}{l}\text { Retroperi- } \\
\text { toneum } \\
\text { (aorta) }\end{array}$ & $\begin{array}{l}\text { Between vena cava } \\
\text { and aorta, } 8 \mathrm{~mm} \text {, } \\
\text { Likert } 4\end{array}$ & $\begin{array}{l}\text { Several small, not } \\
\text { measurable, } \\
\text { Likert } 2\end{array}$ & $\begin{array}{l}\text { CT after } 9 \text { and } 15 \text { months without signs of } \\
\text { retroperitoneal metastasis. }\end{array}$ \\
\hline \multirow[t]{2}{*}{$26 *$} & \multirow[t]{2}{*}{$\mathrm{FN}$} & \multirow[t]{2}{*}{$\begin{array}{l}\text { Retroperi- } \\
\text { toneum } \\
\text { (aorta) }\end{array}$} & \multirow[t]{2}{*}{$\begin{array}{l}\text { Between vena cava } \\
\text { and aorta, } 22 \mathrm{~mm} \text {, } \\
\text { Likert } 4\end{array}$} & $\begin{array}{l}\text { Between vena } \\
\text { cava and aorta, } \\
12 \mathrm{~mm} \text {, Likert } 2\end{array}$ & $\begin{array}{l}\text { CT after } 10 \text { months with additional size } \\
\text { reduction to } 9 \mathrm{~mm} \text {. }\end{array}$ \\
\hline & & & & $\begin{array}{l}\text { No restrictive } \\
\text { diffusion }\end{array}$ & $\begin{array}{l}\text { CT after } 16 \text { and } 27 \text { months without signs } \\
\text { of retroperitoneal metastasis. }\end{array}$ \\
\hline 42 & FP & $\begin{array}{l}\text { Retroperi- } \\
\text { toneum } \\
\text { (aorta) }\end{array}$ & $\begin{array}{l}\text { Left side, } 10 \mathrm{~mm} \text {, } \\
\text { Likert } 4\end{array}$ & $\begin{array}{l}\text { Several small on } \\
\text { left side, not } \\
\text { measurable, } \\
\text { Likert } 1\end{array}$ & $\begin{array}{l}\text { Unchanged on CT after } 9,10,13 \text { and } 17 \\
\text { months }\end{array}$ \\
\hline $58 *$ & FP & $\begin{array}{l}\text { Retroperi- } \\
\text { toneum } \\
\text { (aorta) }\end{array}$ & $\begin{array}{l}\text { In front of vena } \\
\text { cava, } 8 \text { mm, Likert } 3\end{array}$ & $\begin{array}{l}\text { - } \\
\text { (lesion level } \\
\text { comparison not } \\
\text { possible) }\end{array}$ & $\begin{array}{l}\text { CT after } 8 \text { and } 14 \text { months without signs of } \\
\text { retroperitoneal metastasis }\end{array}$ \\
\hline \multirow[t]{2}{*}{61} & \multirow[t]{2}{*}{$\mathrm{FP}$} & \multirow[t]{2}{*}{$\begin{array}{l}\text { Retroperi- } \\
\text { toneum } \\
\text { (aorta) }\end{array}$} & \multirow[t]{2}{*}{$\begin{array}{l}\text { Between vena cava } \\
\text { and aorta, not } \\
\text { measurable, Likert } \\
3\end{array}$} & \multirow[t]{2}{*}{$\begin{array}{l}\text { Between vena } \\
\text { cava and aorta, } 5 \\
\mathrm{~mm} \text {, Likert } 2\end{array}$} & $\begin{array}{l}\text { A persistent } 12 \mathrm{~mm} \text { malignant lymph node } \\
\text { removed after } 5 \text { months. Pathology: } \\
\text { teratoma. }\end{array}$ \\
\hline & & & & & $\begin{array}{l}\text { CT after } 6 \text { and } 11 \text { months without signs of } \\
\text { retroperitoneal metastasis. }\end{array}$ \\
\hline 69 & FN & $\begin{array}{l}\text { Retroperi- } \\
\text { toneum } \\
\text { (aorta) }\end{array}$ & $\begin{array}{l}\text { Right side, } 6 \mathrm{~mm} \text {, } \\
\text { Likert } 4\end{array}$ & $\begin{array}{l}\text { Right side, } 6 \mathrm{~mm} \text {, } \\
\text { Likert } 2\end{array}$ & $\begin{array}{l}\text { Discrete size progression on both CT after } \\
4 \text { and } 7 \text { months, up to } 8 \mathrm{~mm} \text {. No clinical } \\
\text { suspicion of new metastasis. }\end{array}$ \\
\hline 78 & $\mathrm{FP}$ & $\begin{array}{l}\text { Retroperi- } \\
\text { toneum } \\
\text { (aorta) }\end{array}$ & $\begin{array}{l}\text { Left side, } 7 \mathrm{~mm} \text {, } \\
\text { Likert } 3\end{array}$ & $\begin{array}{l}\text { Left side, } 8 \text { mm, } \\
\text { Likert } 1\end{array}$ & $\begin{array}{l}\text { CT after } 6 \text { and } 9 \text { months without signs of } \\
\text { retroperitoneal metastasis. }\end{array}$ \\
\hline 61 & $\mathrm{FN}$ & Groin & $\begin{array}{l}\text { Left side, } 7 \mathrm{~mm} \text {, } \\
\text { Likert } 3\end{array}$ & $\begin{array}{l}\text { Left side, } 10 \text { mm, } \\
\text { Likert } 2\end{array}$ & $\begin{array}{l}\text { CT after } 3,6 \text { and } 11 \text { months without signs } \\
\text { of metastasis in the groin. }\end{array}$ \\
\hline
\end{tabular}

CT described 71 benign lesions (median 1 per patient [IQR 0-5], median size 6 mm [IQR 5-8 mm]) and 256 malignant lesions (median 2 per patient [IQR 0-16], median size $12 \mathrm{~mm}$ [IQR 9-19 mm]). MRI described 106 benign lesions 
(median 2 per patient [IQR 0-6], median size $8 \mathrm{~mm}$ [IQR 6-11 mm]) and 302 malignant lesions (median 3 per patient [IQR 0-19], median size $13 \mathrm{~mm}$ [IQR 10-19 mm]). The majority of malignant lesions were located in the retroperitoneum and lungs (table 4).

Table 4

Number and Likert score of recorded lesions in each region for and post-treatment magnetic resonance imaging (MRI) and post-treatment computed tomography (CT). Likert scale: 1 benign, 2 probably benign, 3 probably malignant, 4 malign. Lesions too small for measurement are not included.

\begin{tabular}{|c|c|c|c|c|c|c|c|c|c|c|c|c|c|c|c|c|}
\hline & \multicolumn{2}{|c|}{ Lung } & \multicolumn{2}{|c|}{$\begin{array}{l}\text { Supra- } \\
\text { clavicular }\end{array}$} & \multicolumn{2}{|c|}{$\begin{array}{l}\text { Media- } \\
\text { stinum }\end{array}$} & \multicolumn{2}{|c|}{$\begin{array}{l}\text { Retroperi- } \\
\text { toneum } \\
\text { (aorta) }\end{array}$} & \multicolumn{2}{|c|}{$\begin{array}{l}\text { Retroperi- } \\
\text { toneum } \\
\text { (iliac) }\end{array}$} & \multicolumn{2}{|c|}{ Groin } & \multicolumn{2}{|c|}{$\begin{array}{l}\text { Other } \\
\text { lymph } \\
\text { nodes }\end{array}$} & \multicolumn{2}{|c|}{ Other } \\
\hline & MRI & CT & MRI & CT & MRI & CT & MRI & CT & MRI & CT & MRI & CT & MRI & CT & MRI & CT \\
\hline 1 & 7 & 15 & 1 & 2 & 12 & 5 & 7 & 5 & 1 & 1 & 4 & 8 & 3 & 6 & 18 & 6 \\
\hline 2 & 5 & 6 & 3 & 1 & 5 & 3 & 13 & 7 & 6 & 1 & 6 & 0 & 9 & 0 & 6 & 5 \\
\hline 3 & 9 & 3 & 5 & 2 & 1 & 1 & 25 & 15 & 5 & 3 & 0 & 2 & 2 & 0 & 2 & 2 \\
\hline 4 & 39 & 30 & 6 & 3 & 17 & 13 & 162 & 157 & 23 & 23 & 3 & 0 & 1 & 0 & 2 & 2 \\
\hline Sum & 60 & 54 & 15 & 8 & 35 & 22 & 207 & 184 & 35 & 28 & 13 & 10 & 15 & 6 & 28 & 15 \\
\hline
\end{tabular}

When comparing pre-treatment scan with post-treatment scan in the same modality, CT had $98 \%$ sensitivity $(95 \% \mathrm{Cl}$ 96-100\%) and 88\% specificity (95\% CI 78-95\%), whereas MRI had 99\% sensitivity (95\% CI 97-100\%) and 78\% specificity (95\% Cl 69-86\%). Details table 2B-C.

The difference between CT and MRI sensitivities was $0.57 \%$ (95\% Cl -1.4-2.5\%) and thus MRI was non-inferior to CT in detecting TC metastasis.

In total, 238 lesions were described on both CT and MRI. The median difference in pre-treatment

size on CT and MRI was $0 \mathrm{~mm}$ [IQR -3-1 mm]. Directly compared to CT on lesion level, MRI had $96 \%$ sensitivity (95\% Cl 94-99\%) and $87 \%$ specificity (95\% Cl 69-92\%). Four lesions were false positive on MRI: a lung lesion and three lymph nodes $\leq 10 \mathrm{~mm}$. Eight lesions were false negative on MRI: a lung lesion, six lymph nodes $\leq 10 \mathrm{~mm}$ and a $12 \mathrm{~mm}$ lymph node without restrictive diffusion. Details table 2D and 3, image examples Fig. 1-2.

In total, 259 lesions were described by one modality only. Different selection in regions with $>5$

lesions accounted for 72 lesions. In general, the remaining lesions were $<10 \mathrm{~mm}$ and located in the retroperitoneum or lungs.

Lung metastases were described by CT in 11 patients and by MRI in 18 patients. On patient level compared to CT, MRI had $82 \%$ sensitivity (95\% Cl 48-98\%) and $88 \%$ specificity (95\% Cl 78-94\%) in detecting lung metastases. In total, 87 lung lesions were described, but only 27 were described by both modalities. On lesion level compared to CT, MRI had $96 \%$ sensitivity (95\% $\mathrm{Cl} 80-94 \%)$ and $50 \%$ specificity (95\% Cl 1.3-99\%) in detecting lung metastases. Details table 2E-F.

In one patient, Th12 invasion of a retroperitoneal conglomerate was only visible on MRI. In another patient, a liver lesion disappeared between the pre- and post-treatment scans. Although described as benign before treatment by both, 
neither CT nor MRI could exclude a liver metastasis. Both findings lead to relevant clinical risk stratification and treatment (image examples Fig. 2).

Eleven patients were operated for residual tumour. In ten patients, the residual tumour was visible on both CT and MRI. In the last patient, a four mm necrotic lesion was described by CT without a corresponding lesion described by MRI (teratoma removed five months after post-treatment scans).

AUC for ROC curves was 0.97 for pre- vs. post-treatment CT, 0.96 for pre- vs. post-treatment MRI and 0.95 for posttreatment MRI vs. post-treatment CT.

The interobserver agreement was substantial between CT and MRI (kappa 0.78).

\section{Discussion}

In this prospective, non-inferiority study, the lesion level sensitivity of MRI was non-inferior to CT. The number of lesions calculated in the power analysis was reached.

On patient level, MRI had $98 \%$ sensitivity and 75\% specificity compared to CT. Disagreement only existed in three patients and concerned lymph nodes of which only one was $>10 \mathrm{~mm}$. On subsequent post-chemotherapy CT, all these lymph nodes diminished in size indicating malignancy (Table 3).

On lesion level using the post-treatment scan as reference within each modality, comparable sensitivities (MRI 99\%, CT 98\%) and slightly differing specificities (MRI 78\%, CT 88\%) were found. Disagreement only existed for 12 lesions in 11 patients (Table 3). MRI and CT performed equally well in detecting residual tumours.

Sensitivity is preferred over specificity from a clinical perspective. Final diagnosis is rarely based on imaging alone but needs confirmation by biopsy or serum tumour markers (DaTeCa 2021). As early diagnosis is vital to prognosis, missing a lesion is much more problematic than raising unwarranted suspicion.

Only few studies have investigated MRI in metastatic TC. Laukka (Laukka et al. 2020) compared MRI with DWI to contrast-enhanced CT in 50 TC patients (47 with retroperitoneal TC). Using clinical data as reference, the sensitivity was comparable (MRI 98\%, CT 96\%), but the specificity differed (MRI 100\%, CT 75\%). Sohaib (Sohaib et al. 2009) studied 52 patients (30 with retroperitoneal TC), and found MRI to have 78-96\% sensitivity and $91-100 \%$ specificity compared to CT. Two other MRI studies included a few patients with metastatic TC but did not calculate diagnostic accuracy (Pfannenberg et al. 2004; Mosavi et al. 2015).

The high MRI sensitivity was in line with our results, however, our MRI specificity was slightly lower.

Due to motion, MRI is usually not well suited for lungs (Khalil et al. 2016). To improve detection of lung lesions, we applied a fast, respiratory-triggered DWIBS b200 sequence. The b-value was high enough to suppress flow from pulmonary veins while obtaining a high SNR. A similar approach has previously been useful in detecting lung metastases in colon cancer (Sivesgaard et al. 2020).

Although only few lung lesions were present, we found MRI to have $96 \%$ sensitivity in detection of lung lesions. This is in line with two metanalyses in which MRI with DWI had 80-83\% sensitivity and $80-93 \%$ specificity in detecting pulmonary nodules (Chen et al. 2013; Li et al. 2014). However, further studies of pulmonary MRI in TC are necessary.

The lack of gold standard e.g. pathology for each lesion was the main limitation. Instead, either CT was used as reference or the combined information from the pre- and post-treatment scans within the same modality was used as 
reference. The assumption was that malignant lesions would shrink or necrotise during the highly effective treatment, whereas benign lesions would appear unchanged. Known limitations would be malignant lesions not responding to treatment (very rare) or reactive lymph nodes shrinking without being malignant (rarely described as malignant).

A major limitation was the lesions described by either, but not both modalities. As an example, most benign lesions (except for benign lymph nodes) do not have increased signal on DWIBS and are not as visible on MRI due to the lower resolution, whereas structures with restrictive diffusion on MRI are not prominent on CT. The different number of benign lesions described created a bias in specificity.

The five largest lesions in each region were recorded, but the readers often disagreed on which were largest. As a result, several of these lymph nodes were only described by one modality.

Knowledge of the diagnosis created a bias towards judging lesions as malignant and thus increasing sensitivity and decreasing specificity.

\section{Conclusions}

In this prospective, non-inferiority study, we found MRI with DWIBS to be non-inferior to contrast-enhanced CT in detecting metastatic TC disease.

It seems realistic that patients with metastatic TC may benefit from non-ionizing MRI follow-up imaging in the future.

\section{Declarations}

Funding

The study was funded by Aarhus University Hospital, Aarhus University and the Health Research Foundation of the Central Denmark Region.

\section{Conflicts of interest}

The authors have no relevant financial or non-financial interests to disclose

\section{Author contribution}

All authors contributed to the study conception and design. Conception and design were performed by Solveig Kärk Abildtrup Larsen, Mads Agerbæk and Erik Morre Pedersen. Data collection was performed by Solveig Kärk Abildtrup Larsen, Vibeke Løgager, Catharina Bylov, Hanne Nellemann, Mads Agerbæk, Anne Birgitte Als and Erik Morre Pedersen. Material preparation and analysis were performed by Solveig Kärk Abildtrup Larsen with supervision of Erik Morre Pedersen. The first draft of the manuscript was written by Solveig Kärk Abildtrup Larsen and all authors commented on previous versions of the manuscript. All authors read and approved the final manuscript.

\section{Ethics approval}

This study was performed in line with the principles of the Declaration of Helsinki. Approval was granted by the Central Denmark Region Ethics Committee (1-10-72-179-17) and the Danish Data Protection Agency (1-16-02-720-17). 


\section{Informed consent}

Informed consent was obtained from all individual participants included in the study. The authors affirm that patients provided informed consent for publication of the images in Fig. 1-2.

\section{References}

1. Chen L, Zhang J, Bao J, et al (2013) Meta-analysis of diffusion-weighted MRI in the differential diagnosis of lung lesions. Journal of magnetic resonance imaging 37:1351-1358. https://doi.org/10.1002/jmri.23939

2. DaTeCa (2021) Testikelkræft - primær screening, udredning, behandling, opfølgning og senfølger

3. Khalil A, Majlath M, Gounant V, et al (2016) Contribution of magnetic resonance imaging in lung cancer imaging. Diagnostic and interventional imaging 97:991-1002. https://doi.org/10.1016/j.diii.2016.08.015

4. Laguna MP, Albers P, Algaba F, et al (2020) EAU Guidelines on Testicular Cancer Edn. presented at the EAU Annual Congress Amsterdam 2020. Arnhem, The Netherlands

5. Larsen SKA, Agerbæk M, Jurik AG, Pedersen EM (2020) Ten years of experience with MRI follow-up of testicular cancer stage I: a retrospective study and an MRI protocol with DWI. Acta oncologica (Stockholm, Sweden) 59:1374-1381. https://doi.org/10.1080/0284186X.2020.1794035

6. Laukka M, Mannisto S, Beule A, et al (2020) Comparison between CT and MRI in detection of metastasis of the retroperitoneum in testicular germ cell tumors: a prospective trial. Acta oncologica 59:1-6. https://doi.org/10.1080/0284186x.2020.1725243

7. Li B, Li Q, Chen C, et al (2014) A Systematic Review and Meta-analysis of the Accuracy of Diffusion-weighted MRI in the Detection of Malignant Pulmonary Nodules and Masses. Academic radiology 21:21-29. https://doi.org/10.1016/j.acra.2013.09.019

8. Liu JP, Hsueh HM, Hsieh E, Chen JJ (2002) Tests for equivalence or non-inferiority for paired binary data. Statistics in Medicine 21:231-245. https://doi.org/10.1002/sim.1012

9. Mosavi F, Laurell A, Ahlström H (2015) Whole body MRI, including diffusion-weighted imaging in follow-up of patients with testicular cancer. Acta Oncologica 54:1763-1769

10. Narine R, Osman H, Wongwaisayawan S, et al (2021) Unenhanced MRI of the abdomen and pelvis for surveillance of patients with stage 1 testicular cancer post-radical orchiectomy. Abdominal Radiology 46:1157-1162. https://doi.org/10.1007/s00261-020-02715-z

11. Norman G (2010) Likert scales, levels of measurement and the "laws" of statistics.(Report). Advances in Health Sciences Education 15:625-632. https://doi.org/10.1007/s10459-010-9222-y

12. Pandharipande P V, Eisenberg JD, Lee RJ, et al (2013) Patients with testicular cancer undergoing CT surveillance demonstrate a pitfall of radiation-induced cancer risk estimates: the timing paradox. Radiology 266:896-904. https://doi.org/10.1148/radiol.12121015

13. Pfannenberg AC, Oechsle K, Bokemeyer C, et al (2004) The role of [18F] FDG-PET, CT/MRI and tumor marker kinetics in the evaluation of postchemotherapy residual masses in metastatic germ cell tumors - Prospects for management. World Journal of Urology 22:132-139. https://doi.org/10.1007/s00345-003-0392-6

14. Pierorazio PM, Cheaib JG, Tema G, et al (2020) Performance Characteristics of Clinical Staging Modalities for Early Stage Testicular Germ Cell Tumors: A Systematic Review. The Journal of urology 203:894-901. https://doi.org/10.1097/JU.0000000000000594

15. Silva M V, Motamedinia P, Badalato GM, et al (2012) Diagnostic Radiation Exposure Risk in a Contemporary Cohort of Male Patients With Germ Cell Tumor. Journal of Urology 187:482-486.

Page $13 / 17$ 
https://doi.org/10.1016/j.juro.2011.10.028

16. Sivesgaard K, Larsen LP, Sørensen M, et al (2020) Whole-body MRI added to gadoxetic acid-enhanced liver MRI for detection of extrahepatic disease in patients considered eligible for hepatic resection and/or local ablation of colorectal cancer liver metastases. Acta radiologica 61:156-167. https://doi.org/10.1177/0284185119855184

17. Sohaib SA, Koh DM, Barbachano Y, et al (2009) Prospective assessment of MRI for imaging retroperitoneal metastases from testicular germ cell tumours. Clinical Radiology 64:362-367. https://doi.org/10.1016/j.crad.2008.10.011

18. Stephenson A, Eggener SE, Bass EB, et al (2019) Diagnosis and Treatment of Early Stage Testicular Cancer: AUA Guideline

19. Su D, Faiena I, Tokarz R, et al (2015) Comparative analysis of the risk of radiation exposure and cost of reduced imaging intensity for surveillance of early-stage nonseminomatous germ cell tumors. Urology 85:141-146. https://doi.org/10.1016/j.urology.2014.09.016

20. SWENOTECA (Swedish and Norwegian Testicular Cancer Group) (2021) SWENOTECA X. A Cancer Care Program for Germ Cell Tumours (including testicular, retroperitoneal and mediastinal tumours)

21. Tarin T V., Sonn G, Shinghal R (2009) Estimating the Risk of Cancer Associated With Imaging Related Radiation During Surveillance for Stage I Testicular Cancer Using Computerized Tomography. Journal of Urology 181:627633. https://doi.org/10.1016/j.juro.2008.10.005

22. The Danish Health and Medicines Authority (2015) Opfølgningsprogram for Testikelkræft

23. Van Walraven C, Fergusson D, Earle C, et al (2011) Association of diagnostic radiation exposure and second abdominal-pelvic malignancies after testicular cancer. Journal of Clinical Oncology 29:2883-2888. https://doi.org/10.1200/JC0.2011.34.6379

\section{Figures}



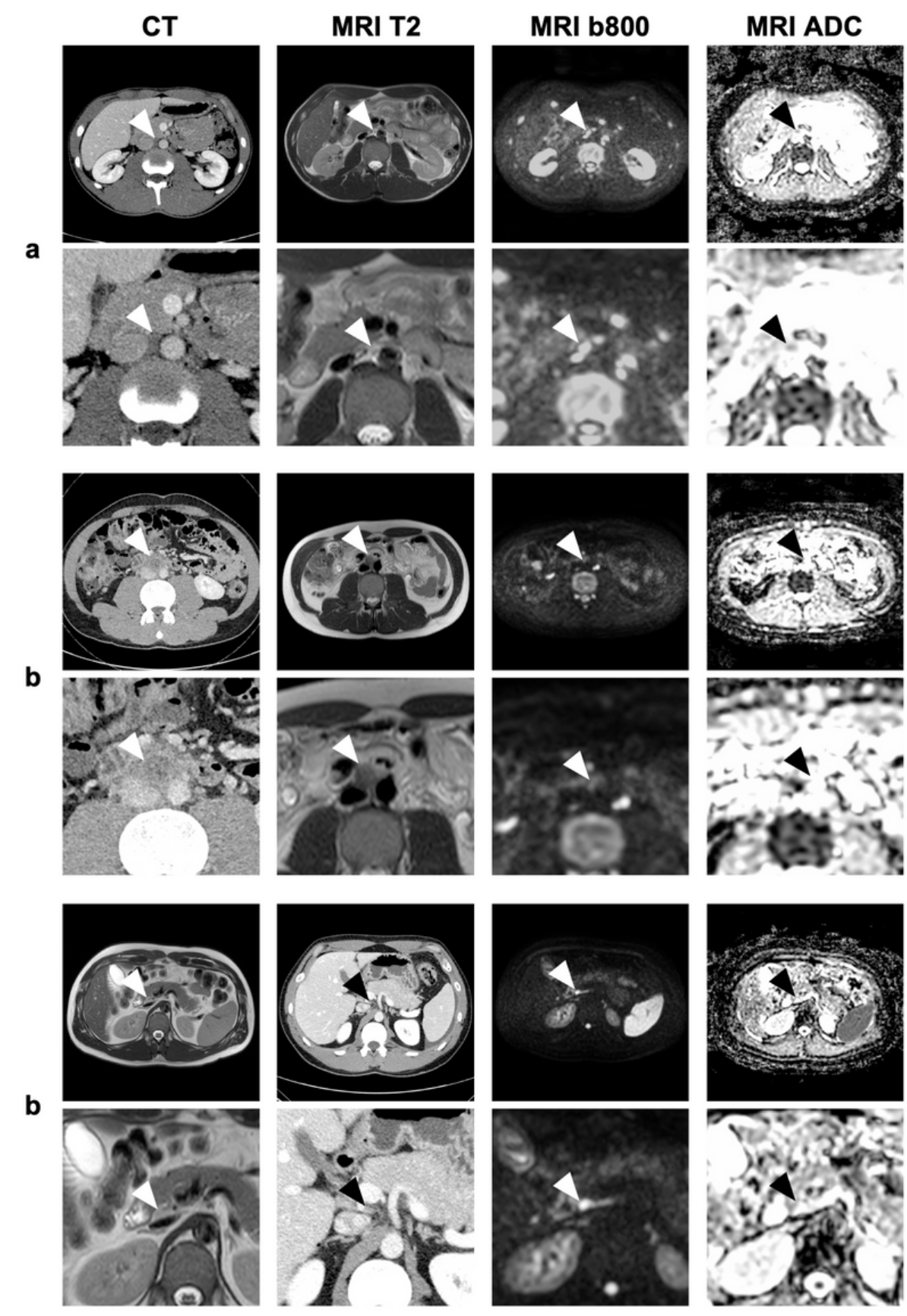

Figure 1

Image examples of false positive and false negative findings on patient level ( $*$ in table 3$)$. Every second line is a zoom-in of the image above. Pre-treatment computed tomography (CT) and pre-treatment magnetic resonance imaging (MRI) (T2, b800 and apparent diffusion coefficient (ADC)).

a: False negative in retroperitoneum: $8 \mathrm{~mm}$ lymph node (Likert 4) on CT. Several small, unmeasurable lymph nodes (Likert 2) on MRI.

b: False negative in retroperitoneum: $22 \mathrm{~mm}$ lymph node (Likert 4) on CT. $12 \mathrm{~mm}$ lymph node without diffusion restriction (Likert 2), almost indistinguishable from the intestines on MRI. 
c: False positive in retroperitoneum: $8 \mathrm{~mm}$ lymph node (Likert 3) on MRI. No lesions described by CT, but a benign lymph node is visible.
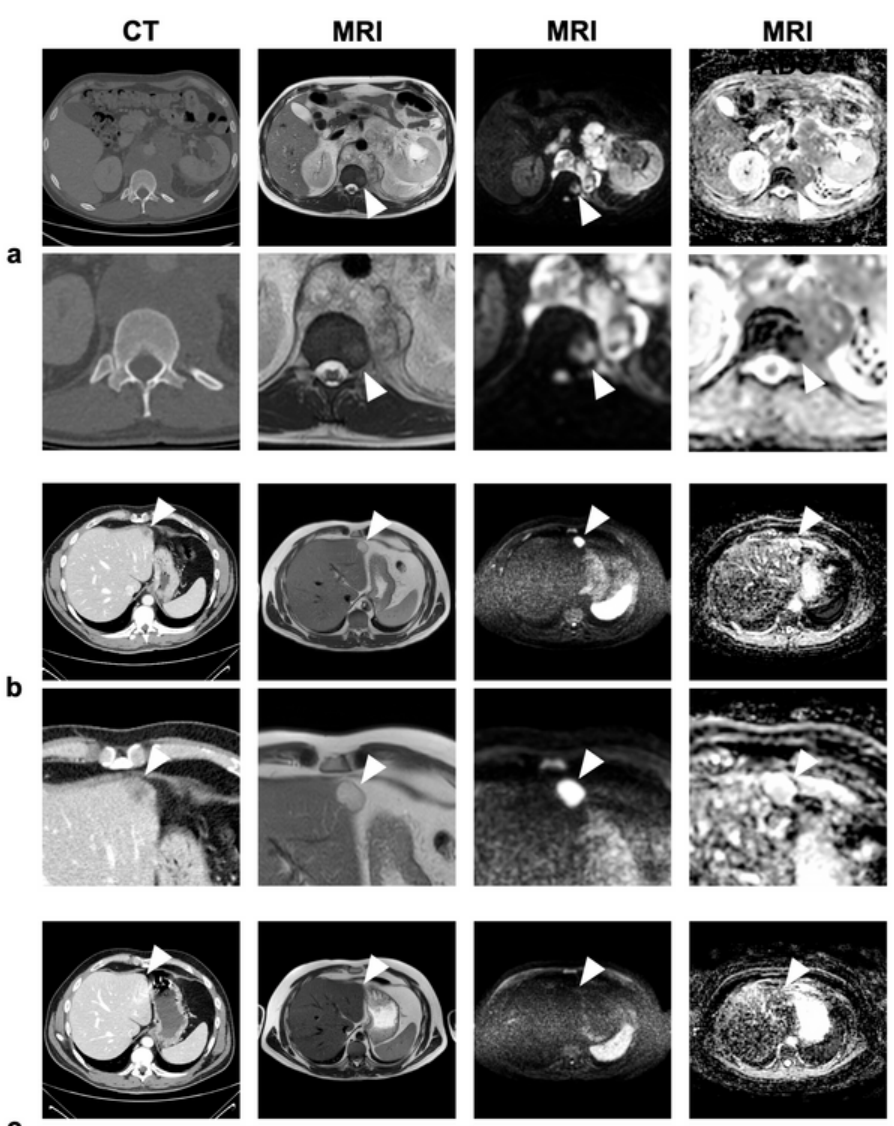

C
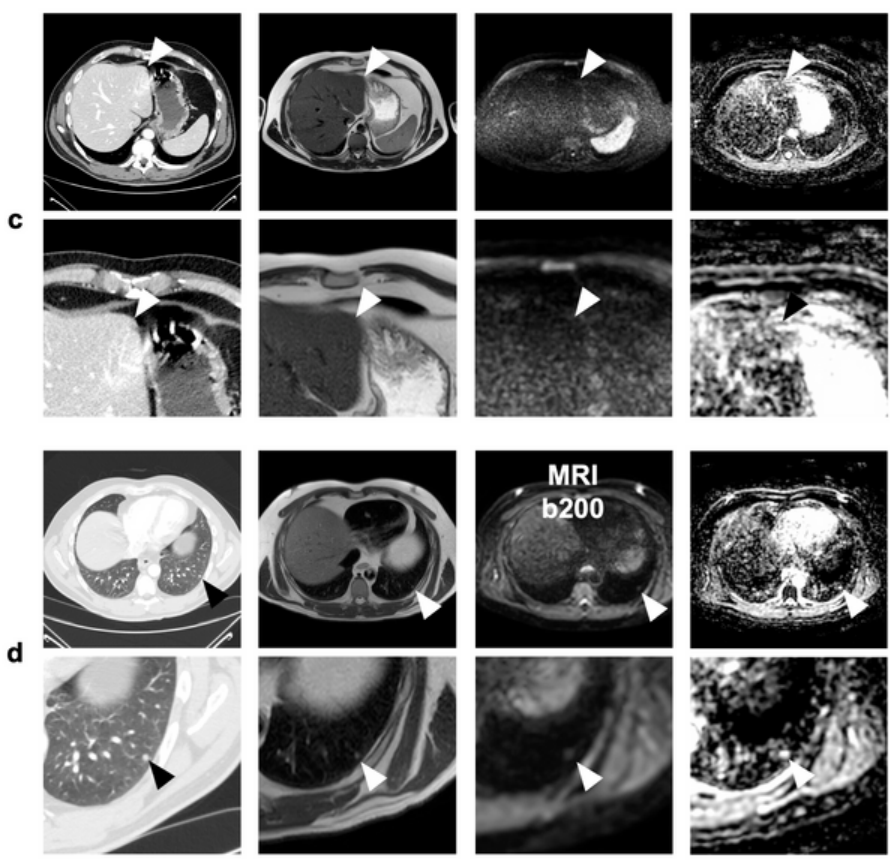

Figure 2

Image examples of lesions in bone, liver and lung, liver and bone on computed tomography (CT) and magnetic resonance imaging (MRI) (T2, b800 and apparent diffusion coefficient (ADC)). Every second line is a zoom-in of the image above.

a (pre-treatment): Bone invasion from retroperitoneal conglomerate into corpus Th12. Only visible on MRI.

b (pre-treatment) and c (post-treatment): Potential liver metastasis. A liver cyst/haemangioma disappearing on both CT and MRI after treatment. 
d (pre-treatment): False negative lung lesion: 5 mm (Likert 4) on CT. 2 mm (Likert 2) on MRI (b200). 